\title{
Modelos para estimativa da eficiência econômica e de estabilidade de uso de nitrogênio na produtividade da aveia
}

\author{
Juliana Aozane da Rosa ${ }^{1}$ \\ Rúbia Diana Mantai ${ }^{2}$ \\ Adriana Roselia Kraisig ${ }^{3}$ \\ José Antonio Gonzalez da Silva ${ }^{4}$ \\ Ivan Ricardo Carvalho 5 \\ Laura Mensch Pereira ${ }^{6}$ \\ Vanessa Pansera ${ }^{7}$ \\ Odenis Alessi ${ }^{8}$ \\ Departamento de Ciências Exatas e Engenharias e Departamento de Estudos Agrários, UNIJUí, Ijuí, RS
}

\begin{abstract}
Resumo. Modelos matemáticos aplicados em biossistemas trazem informações relevantes para o planejamento de práticas agrícolas, viabilizando sistemas de produção sustentáveis e inovadores. Para alcançar a máxima expressão de produtividade dos grãos de aveia é necessário o ajuste de técnicas de manejo que proporcionem melhor eficiência de uso de nitrogênio pela planta. Neste contexto, o objetivo do estudo é estabelecer a estimativa para a otimização da capacidade de aproveitamento de nitrogênio pela aveia à maior produtividade de grãos e de indústria por modelos que dimensionam a máxima eficiência técnica, econômica e de estabilidade. O estudo foi realizado em delineamento de blocos casualizados com quatro repetições seguindo um modelo fatorial 4 x 2 , para doses de nitrogênio $\left(0,30,60\right.$ e $\left.120 \mathrm{~kg} \mathrm{ha}^{-1}\right)$ e cultivares de aveia (Barbarasul e Brisasul), no sistema de sucessão soja/aveia. A máxima eficiência técnica, independente da condição de ano agrícola promove maior produtividade de grãos e de indústria com as doses médias de 86 e $81 \mathrm{~kg} \mathrm{ha}^{-1}$ de nitrogênio, respectivamente. Já, a máxima eficiência econômica, considerando a relação preço do produto e insumo, independente da condição de ano agrícola proporcionou valores similares da produtividade de grãos e de indústria aos obtidos pela máxima eficiência técnica com as doses médias de $71 \mathrm{~kg} \mathrm{ha}^{-1}$ e $51 \mathrm{~kg} \mathrm{ha}^{-1}$ de nitrogênio. O parâmetro de estabilidade indica comportamento previsível, independente da condição de ano agrícola na dose de $60 \mathrm{~kg} \mathrm{ha}^{-1}$ de nitrogênio para a produtividade de grãos e de indústria. Esta dose de nitrogênio promove, alta produtividade e qualidade de grãos, aliada a possibilidade de diminuir as perdas de nitrogênio ao ambiente, reduzindo os impactos ambientais.
\end{abstract}

Palavras-chave. Avena sativa, Máxima Eficiência Econômica, Máxima Eficiência de Estabilidade, Modelagem Matemática, Sustentabilidade

\footnotetext{
1 juaozane@gmail.com

${ }^{2}$ rdmantai@yahoo.com.br

3 maryshelei@yahoo.com.br

4 jagsfaem@yahoo.com.br

5 carvalho.irc@gmail.com

${ }^{6}$ lauramensch@gmail.com

${ }^{7}$ vpansera@hotmail.com

8 odenisalessi@hotmail.com
} 


\section{Introdução}

A modelagem matemática é uma ferramenta utilizada para explicar e interpretar fatos e fenômenos observados de diversas áreas do conhecimento, identificando características, realizando previsões e mostrando tendências ao longo do desenvolvimento. Assim, existe a necessidade de otimizar a produção de alimentos a partir de tecnologias que garantam produtividade com redução de custos e sustentabilidade aos agroecossistemas $[4,9]$.

Para a máxima expressão do potencial de produtividade dos grãos de aveia, é necessário o ajuste de técnicas de manejo, sendo a disponibilidade de nitrogênio decisiva em proporcionar efeitos significativos na produtividade e qualidade de grãos de aveia [2,10]. Na expectativa de altos rendimentos eleva-se a quantidade de nitrogênio, porém, dependendo das condições ambientais, a eficiência de uso do nutriente é reduzida, diminuindo consideravelmente a produtividade, aumentando os custos de produção e gerando poluição ambiental, seja por lixiviação do nitrato ou volatilização da amônia [1,2]. Assim, a forte variação da produtividade está associada à variabilidade das condições meteorológicas, de modo que, anos favoráveis e desfavoráveis ao cultivo alteram a disponibilidade de nitrogênio e a eficiência de uso pela planta, implicando em uma maior instabilidade de produtividade $[2,5]$.

Estudos que visam melhorar a eficiência de uso do nitrogênio podem qualificar as recomendações e viabilizar tecnologias mais ajustadas. O uso de equações de regressão são satisfatoriamente empregados no estudo de processos em biossistemas, descrevendo fenômenos e possibilitando a realização de simulações e otimizações $[11,12]$. O modelo de regressão polinomial é utilizado na determinação da eficiência técnica, definindo a dose de nitrogênio que otimiza a variável de interesse sem considerar a viabilidade econômica. Por outro lado, o uso de regressão polinomial envolvendo o preço do insumo e do produto permite otimizar a dose ótima considerando a eficiência econômica $[13,14]$. Os modelos de regressão envolvendo parâmetros de estabilidade, podem identificar processos mais estáveis e eficientes na variável de interesse, com comportamento previsível independente das condições meteorológicas dos anos agrícolas.

O objetivo do estudo é estabelecer a estimativa para a otimização da capacidade de aproveitamento de nitrogênio pela aveia à maior produtividade de grãos e de indústria por modelos que dimensionam a máxima eficiência técnica, econômica e de estabilidade.

\section{Material e Métodos}

O experimento foi conduzido a campo, em Augusto Pestana, RS, Brasil. O delineamento experimental foi o de blocos casualizados com quatro repetições, seguindo um esquema fatorial $4 \mathrm{x}$ 2, para doses de nitrogênio $\left(0,30,60\right.$ e $\left.120 \mathrm{~kg} \mathrm{ha}^{-1}\right)$ e cultivares de aveia (Barbarasul e Brisasul), totalizando 32 unidades experimentais no sistema de sucessão soja/aveia. A produtividade de grãos foi obtida pelo corte de três linhas centrais de cada parcela no estádio de maturidade de colheita, com umidade de grãos ao redor de $22 \%$. As plantas foram trilhadas com colheitadeira estacionária e direcionadas ao laboratório para correção da umidade de grãos para 13\%; e pesagem para estimativa da produtividade de grãos $\left(\mathrm{PG}, \mathrm{kg} \mathrm{ha}^{-1}\right)$ e de indústria $\left(\mathrm{PI}, \mathrm{kg} \mathrm{ha}^{-1}\right)$.

Ao atender aos pressupostos de homogeneidade e normalidade via testes de Bartlett, foi realizada análise de variância para detecção dos efeitos principais e de interação. Através do modelo de Scott e Knott foi realizado o teste de comparação de médias agrupando doses de adubação nitrogenada que respondem de forma homogênea a variável em estudo, classificando-as por eficiência, independente de ano agrícola. Posteriormente, foi realizado o modelo de Eberhart e Russel, baseando-se em uma regressão linear com a inclusão do índice ambiental codificado, para análise de estabilidade independente de ano agrícola. A estabilidade da dose de aplicação do nitrogênio foi obtida pelo parâmetros $S_{i j}^{2}$, sendo que uma condição é considerada estável quando $S_{i j}^{2}=0$ e instável quando 
$S_{i j}^{2} \neq 0$. Sucedeu-se também o ajuste da regressão quadrática para estimativa da máxima eficiência econômica da produtividade de grãos e de indústria. Para a máxima eficiência econômica estão incluídos no modelo o preço do produto $(\mathrm{w})$ e o preço do insumo $(\mathrm{t})$. Os valores utilizados representam os preços médios comercializados em dezembro de 2018, sendo o preço do insumo (ureia) de $\mathrm{R} \$ 1,25 \mathrm{~kg}^{-1}, \mathrm{R} \$ 2,80 \mathrm{~kg}^{-1}$ do nitrogênio, e do produto aveia de $\mathrm{R} \$ 0,60 \mathrm{~kg}^{-1}$.

A máxima eficiência econômica foi determinada através da equação quadrática (1), sendo que a variável independente representa o produto avaliado, neste caso, a quantidade de nitrogênio e a variável dependente a produtividade de grãos e de indústria.

$$
y=b_{0}+b_{1} x+b_{2} x^{2}+\epsilon
$$

Onde y $=$ variável dependente, $\mathrm{x}$ a variável independente, $\epsilon$ o erro aleatório, $b_{0}, b_{1}$ e $b_{2}$ são parâmetros a serem estimados com a utilização do método dos mínimos quadrados.

O ponto máximo de uma curva, ou seja, a produtividade máxima alcançada é obtida pela derivada da função quadrática homogênea (2):

$$
y^{\prime}=b_{1}+2 b_{2} x
$$

Entretanto, o valor gasto para atingir o pico de produção pod não cobrir a despesa do insumo utilizado. Nesse caso, as variáveis x e y poderão ter pesos t e w, respecivamente diferentes, sendo t o preço do insumo e w o preço do produto [14]. Assim o modelo da máxima eficiência econômica é dado por (3):

$$
x=\left(\frac{t}{w}-b_{1}\right) /\left(2 b_{2}\right)(3)
$$

Para a máxima eficiência técnica da produtividade de grãos e de indústria, também foi utilizada a equação quadrática (1), sendo x a quantidade de nitrogênio aplicado, e y a produtividade de grãos. Assim, a máxima eficiência técnica foi obtida pela derivada da função quadrática homogênea (4) [14]. Deste modo, x representa a dose de nitrogênio que implica na maior produtividade y, ou seja, a máxima eficiência técnica.

$$
x=\frac{-b_{1}}{2 b_{2}}
$$

Os procedimentos de testes de médias, estabilidade por Eberhart e Russell, análise de regressão linear e quadrática, foram realizadas com o auxílio do software GENES.

\section{Resultados e Discussão}

Na Tabela 1 do sistema soja/aveia, as médias e parâmetros de estabilidade a maior produtividade de grãos foi obtida nas doses mais elevadas de nitrogênio. Além disso, o parâmetro de estabilidade do modelo indicou comportamento previsível, independente da condição de ano agrícola, na dose de $60 \mathrm{~kg} \mathrm{ha}^{-1}$ de nitrogênio. $\mathrm{Na}$ análise da produtividade de indústria esta mesma condição foi observada, destacando a mais elevada média de produtividade com estabilidade também na dose de $60 \mathrm{~kg} \mathrm{ha}^{-1}$ do nutriente. Assim, a dose de $60 \mathrm{~kg} \mathrm{ha}^{-1}$ de nitrogênio evidencia estabilidade, de recomendação independente da condição de ano agrícola nesta espécie.

Na Tabela 2 do sistema soja/aveia, a análise das eficiências através de regressões quadráticas, destaca-se que em todas as equações foi observado que os pontos analisados estavam dentro do intervalo de confiança das distintas observações. Nesta perspectiva, utilizando a eficiência econômica considerando a relação preço do produto e insumo, a eficiência econômica reduziu em mais de $13 \mathrm{~kg} \mathrm{ha}^{-1}$ a quantidade de nitrogênio em ano favorável, e de $26 \mathrm{~kg} \mathrm{ha}^{-1}$ de nitrogênio no ano 
Tabela 1: Valores médios e parâmetros de estabilidade das variáveis de produtividade de grãos e de indústria em distintas doses de nitrogênio no sistema soja/aveia.

\begin{tabular}{|c|c|c|c|c|c|c|c|c|c|c|}
\hline \multirow[t]{2}{*}{$\mathrm{Y}$} & \multirow[t]{2}{*}{$\begin{array}{c}\text { Dose N } \\
\left(k g h a^{-1}\right)\end{array}$} & \multicolumn{6}{|c|}{ Anos } & \multirow[t]{2}{*}{$\bar{y}$} & \multicolumn{2}{|c|}{$\begin{array}{c}\text { Parâmetros } \\
\text { do Modelo }\end{array}$} \\
\hline & & $\begin{array}{l}2011 \\
(\mathrm{AF})\end{array}$ & $\begin{array}{l}2012 \\
(\mathrm{AD})\end{array}$ & $\begin{array}{l}2013 \\
(\mathrm{AF})\end{array}$ & $\begin{array}{l}2014 \\
(\mathrm{AD})\end{array}$ & $\begin{array}{l}2015 \\
(\mathrm{AI})\end{array}$ & $\begin{array}{l}2016 \\
(\mathrm{AI})\end{array}$ & & $S_{i j}^{2}$ & $R^{2}$ \\
\hline & 0 & 2989 & 1745 & 3036 & 1645 & 2746 & 2461 & 2437 c & $2882^{n s}$ & 98 \\
\hline PG & 30 & 3694 & 2361 & 3721 & 2132 & 3361 & 3279 & $3091 \mathrm{~b}$ & $-4629 n s$ & 99 \\
\hline \multirow[t]{4}{*}{$\left(\operatorname{kg} h a^{-1}\right)$} & 60 & 4124 & 2751 & 4174 & 2426 & 3825 & 3884 & 3531 a & $367^{n s}$ & 99 \\
\hline & 120 & 3938 & 2654 & 3994 & 2522 & 3871 & 3717 & 3449 a & $-211 *$ & 98 \\
\hline & $\bar{x}$ & 3686 & 2378 & 3731 & 2181 & 3451 & 3335 & 3127 & & \\
\hline & 0 & 1109 & 1037 & 1504 & 891 & 1098 & 315 & $992 \mathrm{c}$ & $-317^{n s}$ & $\overline{98}$ \\
\hline PI & 30 & 1578 & 1440 & 1810 & 1100 & 1453 & 403 & $1297 \mathrm{~b}$ & $2273^{n s}$ & 98 \\
\hline \multirow[t]{3}{*}{$\left(\operatorname{kg} h a^{-1}\right)$} & 60 & 1698 & 1665 & 2169 & 1294 & 1701 & 424 & 1492 a & $-1161^{n s}$ & 99 \\
\hline & 120 & 1560 & 1689 & 2108 & 1393 & 1442 & 355 & $1425 \mathrm{a}$ & $7577^{*}$ & 97 \\
\hline & $\bar{x}$ & 1486 & 1458 & 1898 & 1170 & 1424 & 374 & 1302 & & \\
\hline
\end{tabular}

$\overline{\mathrm{PG}}=$ Produtividade de grãos; $\mathrm{PI}=$ Produtividade industrial; $\mathrm{AF}=$ Ano favorável; $\mathrm{AD}=$ Ano desfavorável; $\mathrm{AI}=\mathrm{Ano}$ intermediário; $\bar{y}=$ Valores médios em relação a dose de nitrogênio; $\bar{x}=$ Valores médios em relação ao ano de cultivo; $S_{i j}^{2}=$ Desvio padrão da regressão; $R^{2}=$ Coeficiente de determinação (\%). Médias seguidas de mesma letra não diferem entre si pelo teste de Skott-Knott em nível de $5 \%$ de probabilidade de erro. ${ }^{*}=$ Significativo a $5 \%$ de probabilidade de erro pelo teste $\mathrm{F} ; \mathrm{ns}=$ não significativo.

desfavorável de 2014, mantendo valores similares de produtividade de grãos. Foi considerado para estimativa de produtividade, a dose de estabilidade obtida na Tabela 1, de $60 \mathrm{~kg} \mathrm{ha}^{-1}$ de nitrogênio. Em todas as condições de ano agrícola, houve uma redução entre 20 e $9 \mathrm{~kg} \mathrm{ha}^{-1}$ do nutriente aplicado, considerando a máxima eficiência técnica e econômica de produtividade, respectivamente, mostrando valores similares de produtividade de grãos. Além disso, os resultados obtidos pela máxima eficiência técnica, econômica e de estabilidade, independente da condição de ano agrícola, evidenciam médias similares de produtividade. Um resultado altamente relevante, que incide em colocar menos insumos e poluir menos o ambiente, em anos nos quais a eficiência de uso do nutriente é reduzida, além de manter a elevada produtividade de grãos em anos favoráveis ao aproveitamento do nitrogênio.

Destaca-se que em anos favoráveis, a máxima eficiência técnica de uso do nitrogênio à produtividade de indústria foi atingida com doses menores em comparação com a produtividade de grãos, alcançando uma média de $1955 \mathrm{~kg} \mathrm{ha}{ }^{-1}$ com dose em torno de $78 \mathrm{~kg} \mathrm{ha}^{-1}$ de nitrogênio. Em anos desfavoráveis, a máxima expressão foi alcançada com uma dose de nitrogênio ao redor de 104 $\mathrm{kg} \mathrm{ha} \mathrm{h}^{-1}$, garantindo uma produtividade de indústria ao redor de $1590 \mathrm{~kg} \mathrm{ha}^{-1}$. Estes resultados mostram a importância da influência dos fatores meteorológicos para a indicação da adubação nitrogenada, de modo que, a variação de ano favorável para desfavorável implica $33 \%$ de aumento de dose do nutriente e ainda redução de $19 \%$ da produtividade industrial. O uso de nitrogênio pela máxima eficiência econômica reduziu a dose do nutriente até $24 \mathrm{~kg} \mathrm{ha}^{-1} \mathrm{em}$ ano favorável e 43 $\mathrm{kg} \mathrm{ha}^{-1}$ em ano desfavorável. Portanto, reduções significativas de nitrogênio com produtividades próximas à máxima eficiência técnica. Na análise de uso do nitrogênio por modelos que dimensionam comportamento previsível, a máxima eficiência de estabilidade com produtividade industrial mais elevada foi obtida com $60 \mathrm{~kg} \mathrm{ha}{ }^{-1}$ de nitrogênio. Uma condição que reduziu fortemente o uso do nutriente em relação a máxima eficiência técnica e econômica, mantendo produtividades similares. Esta condição possibilitou reduções do nutriente de até $51 \mathrm{~kg} \mathrm{ha}^{-1}$ em comparação a máxima eficiência técnica, com queda máxima de produtividade de indústria de $112 \mathrm{~kg} \mathrm{ha}^{-1}$. Os 
Tabela 2: Estimativa da máxima eficiência econômica e de estabilidade pelo nitrogênio à simulação de produtividade de grãos e de indústria no sistema soja/aveia.

\begin{tabular}{|c|c|c|c|c|c|c|}
\hline Ano & $\left(y=b_{0}+b_{1} x+b_{2} x^{2}\right)$ & $R^{2}$ & $N_{M E E}$ & $\begin{array}{c}P G_{M E E} \\
\left(\operatorname{kg} h a^{-1}\right)\end{array}$ & $N_{M E S}$ & $\begin{array}{l}P G_{M E S} \\
\left(\mathrm{~kg} h a^{-1}\right)\end{array}$ \\
\hline \multicolumn{7}{|c|}{ Produtividade de grãos } \\
\hline $2011(\mathrm{AF})$ & $2983+29,6 \mathrm{x}-0,18^{*} x^{2}$ & 99 & 69 & 4168 & \multirow{6}{*}{60} & 4111 \\
\hline $2012(\mathrm{AD})$ & $1739+25,7 \mathrm{x}-0,15^{*} \mathrm{x} x^{2}$ & 99 & 70 & 2801 & & 2741 \\
\hline $2013(\mathrm{AF})$ & $3025+29,5 \mathrm{x}-0,18^{*} x^{2}$ & 99 & 69 & 4202 & & 4147 \\
\hline $2014(\mathrm{AD})$ & $1647+18,8 \mathrm{x}-0,09^{*} x^{2}$ & 99 & 78 & 2565 & & 2451 \\
\hline $2015(\mathrm{AI})$ & $2734+26,1 \mathrm{x}-0,14^{*} x^{2}$ & 99 & 76 & 3909 & & 3796 \\
\hline $2016(\mathrm{AI})$ & $2440+36,1 \mathrm{x}-0,21^{*} x^{2}$ & 99 & 74 & 3964 & & 3850 \\
\hline $\bar{x}$ & $2428+27,6 \mathrm{x}-0,16^{*} x^{2}$ & 99 & 71 & $3582 \mathrm{a}$ & 60 & $3508 \mathrm{a}$ \\
\hline \multicolumn{7}{|c|}{ Produtividade de indústria } \\
\hline $2011(\mathrm{AF})$ & $1128+16,7 \mathrm{x}-0,1^{*} x^{2}$ & 98 & 60 & 1768 & \multirow{6}{*}{60} & 1734 \\
\hline $2012(\mathrm{AD})$ & $1040+15,6 \mathrm{x}-0,1^{*} x^{2}$ & 99 & 54 & 1591 & & 1688 \\
\hline $2013(\mathrm{AF})$ & $1478+16,0 \mathrm{x}-0,1^{n s} \mathrm{x}^{2}$ & 97 & 56 & 2060 & & 2078 \\
\hline $2014(\mathrm{AD})$ & $884+8,9 \mathrm{x}-0,04^{n s} \mathrm{x}^{2}$ & 99 & 51 & 1235 & & 1274 \\
\hline $2015(\mathrm{AI})$ & $1086+16,7 \mathrm{x}-0,1^{*} x^{2}$ & 99 & 60 & 1726 & & 1692 \\
\hline 2016 (AI) & $317+3,4 \mathrm{x}-0,03^{n s} \mathrm{x}^{2}$ & 98 & 00 & 317 & & 413 \\
\hline $\bar{x}$ & $985+12,9 \mathrm{x}-0,08^{n s} \mathrm{x}^{2}$ & 99 & 51 & $1433 \mathrm{a}$ & 60 & $1475 \mathrm{a}$ \\
\hline
\end{tabular}

$R^{2}=$ Coeficiente de determinação $(\%) ; N_{M E E}=$ Dose de nitrogênio à máxima eficiência econômica; $P G_{M E E}=$ Produtividade de grãos pela máxima eficiência econômica; $N_{M E S}=$ Dose de nitrogênio à máxima eficiência de estabilidade; $P G_{M E E}=$ Produtividade de grãos pela máxima eficiência de estabilidade; $\mathrm{AF}=$ Ano favorável; $\mathrm{AD}=\mathrm{Ano}$ desfavorável; $\mathrm{AI}=$ Ano intermediário; ${ }^{*}=$ Significativo a $5 \%$ de probabilidade de erro, pelo teste $\mathrm{t} ;{ }^{n s}=$ não significativo; Médias seguidas de mesma letra não diferem entre si pelos teste de Scott e Knott em nível de $5 \%$ de probabilidade de erro.

resultados obtidos evidenciam a possibilidade de propostas de otimização de uso do nitrogênio levando em consideração a análise do custo do produto e do insumo e as tendências meteorológicas do ano de cultivo, visto forte interação com a eficiência de aproveitamento do nutriente.

O manejo do nitrogênio tem sido uma das práticas agrícolas mais estudadas, com o intuito de melhorar a eficiência de uso, diminuir os custos de produção, poluição ambiental e incrementar a produtividade. A dose de nitrogênio pela máxima eficiência econômica deve ser considerada observando as tendências meteorológicas do ano de cultivo [7]. Em trigo, a produtividade de grãos pelo dimensionamento da dose de nitrogênio, determinou reduções significativas na quantidade do nutriente pela eficiência econômica com produtividades similares as obtidas pela máxima eficiência técnica [2]. Em milho, atingiram a máxima produtividade de grãos com a dose de $289 \mathrm{~kg} \mathrm{ha}^{-1}$ de nitrogênio, porém com maior eficiência econômica com $157 \mathrm{~kg}$ ha ${ }^{-1}$. Portanto, concluíram que a máxima produtividade de grãos pela eficiência técnica não resulta em melhores rendimentos num contexto geral sobre a produção [8]. Fato também observado em cereais como arroz [6]. A aveia é altamente influenciada pelas condições de ambiente, portanto métodos que visam caracterizar a adaptabilidade e estabilidade que se baseiam nas interações entre genótipos e ambiente são úteis na definição de tecnologias de manejo mais estáveis e eficientes nos sistemas de cultivo [3].

\section{Conclusões}

A máxima eficiência técnica, independente da condição de ano agrícola promove maior produtividade de grãos e de indústria com as doses médias de 86 e $81 \mathrm{~kg} \mathrm{ha}^{-1}$ de nitrogênio, respectivamente. Já, a máxima eficiência econômica, considerando a relação preço do produto e insumo, independente da condição de ano agrícola proporcionou valores similares da produtividade de grãos 
e de indústria aos obtidos pela máxima eficiência técnica com as doses médias de $71 \mathrm{~kg} \mathrm{ha}^{-1} \mathrm{e} 51 \mathrm{~kg}$ $\mathrm{ha}^{-1}$ de nitrogênio. O parâmetro de estabilidade indica comportamento previsível, independente da condição de ano agrícola na dose de $60 \mathrm{~kg} \mathrm{ha}^{-1}$ de nitrogênio para a produtividade de grãos e de indústria. Esta dose de nitrogênio promove, alta produtividade e qualidade de grãos, aliada a possibilidade de diminuir as perdas de nitrogênio ao ambiente, reduzindo os impactos ambientais.

\section{Referências}

[1] Arenhardt, E. G., Da Silva, J. A. G., Gewehr, E., De OLiveira, A. C., Binelo, M. O., Valdiero, A. C., Gzergorczick, M. E., De Lima, A. R. C. O suprimento de nitrogênio no cultivo de trigo depende das condições climáticas e do sistema de sucessão no sul do Brasil. Revista Africana de Pesquisa Agrícola, volume 10, p. 4322-4330, 2015. DOI: 10.5897/AJAR2015.10038.

[2] Da Silva, J. A. G., Goi Neto, C. J., Fernandes, S. B. V., Mantai, R. D., Scremin, O. B., Pretto, R. Eficiência de nitrogênio em aveia no rendimento de grãos com estabilidade. Revista Brasileira de Engenharia Agrícola e Ambiental, volume 20, p. 1095-1100, 2016. DOI: 10.1590/1807-1929/agriambi.v20n12p1095-1100.

[3] Da Silva, J. A. G., Wohlenberg, M. D., Arenhardt, E. G., De Oliveira, A. C., Mazurkievicz, G., Muller, M., Arenhardt, L. G., Binelo, M. O., Arnold, G., Pretto, R. Adaptability and stability of yield and industrial grain quality with and without fungicide in Brazilian oat cultivars. American Journal of Plant Sciences, volume 6, p.1560-1569, 2015. DOI: 10.4236/ajps.2015.69155.

[4] De Mamann, A. T. W., da Silva, J. A. G, Binelo, M. O., Scremin, O. B., Kraisig, A. R., Carvalho, I. R., Argenta, C. V. Inteligência Artificial Simulando Produtividade de Grãos Durante o Desenvolvimento do Trigo Considerando Indicadores Biológicos e Ambientais. Journal of Agricultural Studies, volume 7, p. 197-212, 2019. ISSN: 2166-0379.

[5] Espindula, M. C., Rocha, V. S., Souza, M. A. D., Grossi, J. A. S., Souza, L. T. D. Doses e formas de aplicação de nitrogênio no desenvolvimento e produção da cultura do trigo. Ciência e Agrotecnologia, volume 34, p. 1404-1411, 2010. DOI: 10.1590/S1413-70542010000600007.

[6] Freitas, T. F. S., Silva, P. R. F. D., Mariot, C. H. P., Menezes, V. G., Anghinoni, I., Bredemeier, C., Vieira, V. M. Produtividade de arroz irrigado e eficiência da adubação nitrogenada influenciadas pela época da semeadura. Revista Brasileira de Ciência do Solo, volume 32, p. 2397-2405, 2008. DOI: 10.1590/S0100-06832008000600018.

[7] Hawerroth, M. C., Da Silva, J. A. G., Woymann, L. G., Zimmer, C. M., Groli, E. L., De Oliveira, A. C., De Carvalho, F. I. F. Correlations among industrial traits in oat cultivars grown in different locations of Brazil. Australian Journal of Crop Science, volume 9, p. 11821189, 2015. ISSN: 1835-2693.

[8] Pavinato, P. S., Ceretta, C. A., Girotto, E., Moreira, I. C. L. Nitrogênio e potássio em milho irrigado: análise técnica e econômica da fertilização. Ciência Rural, volume 38, p. 358-364, 2008. DOI: $10.1590 /$ S0103-84782008000200010.

[9] Sala, V. M. R., Freitas, S. D. S., Donzeli, V. P., Freitas, J. G., Gallo, P. B., Silveira, A. P. D. D. Ocorrência e efeito de bactérias diazotróficas em genótipos de trigo. Revista Brasileira de Ciência do Solo, volume 29, p. 345-352, 2005. DOI:10.1590/S0100-06832005000300004.

[10] Scremin, O. B., Da Silva, J. A. G., De Mamann, A. T. W., Mantai, R. D., Brezolin, A. P., Marolli, A. Eficiência de nitrogênio no rendimento de aveia através do biopolímero hidrogel. 
Revista Brasileira de Engenharia Agrícola e Ambiental, volume 21, p. 379-385, 2017. DOI: 10.1590/1807-1929/agriambi.v21n6p379-385.

[11] Seffrin, R., Araújo, E. C. de., Bazzi, C. L. Regression models for prediction of corn yield in the state of Paraná (Brazil) from 2012 to 2014. Acta Scientiarum. Agronomy, volume 40, p.1-11, 2018. DOI: 10.4025 /actasciagron.v40i1.36494.

[12] Tavares, L. C., Rufino, C. D. A., Brunes, A. P., Tunes, L. M. D., Barros, A. C. S. A., Peske, S. T. Desempenho de sementes de soja sob deficiência hídrica: rendimento e qualidade fisiológica da geração F1. Ciência Rural, volume 43, p. 1357-1363, 2013. DOI: 10.1590/S010384782013000800003

[13] Vieira, F. A., Bezerra Neto, F., Silva, M. L. D., LIMA, J. S. S. D., Barros Junior, A. P., Silva, I. N. Technical-economic efficiency of the yield of green grains of cowpea fertilized with roostertree. Revista Caatinga, volume 31, p. 504-510, 2018. DOI: 10.1590/1983-21252018v31n227rc.

[14] Wendling, A., Eltz, F. L. F., Cubilla, M. M., Amado, T. J. C., Mielniczuk, J., Lovato, T. Recomendação de adubação nitrogenada para trigo em sucessão ao silho e soja sob sistema plantio direto no Paraguai. Revista Brasileira de Ciência do Solo, volume 31, p. 985-994, 2007. DOI: 10.1590/S0100-06832007000500015. 